\title{
Synthesis and characterization of graphene oxide-molecularly imprinted polymer for Neopterin adsorption study
}

\begin{abstract}
Neopterin (NEO) is a useful biomarker for detection of malignant diseases. However, adsorption study using graphene oxide-molecularly imprinted polymer (GO-MIP) material has been lacking. The aim of this research was to synthesize and characterize GO-MIP for use in NEO adsorption study. GO-MIP with NEO as the template was synthesized via free radical polymerization method, with methacrylic acid (MAA) as the monomer, ethylene glycol dimethacrylate (EGDMA) as the cross-linker, ammonium persulfate (APS) as the initiator, and $8 / 2 \mathrm{v} / \mathrm{v}$ ratio of dimethylsulfoxide/acetonitrile (DMSO/ACN) solution as porogen solvent. The formation of GO-MIP hybrid with NEO binding sites was verified via Fourier transform infrared spectroscopy (FTIR), CHNS analysis, thermogravimetric analysis (TGA), field emission scanning electron microscopy (FESEM), and transmission electron microscopy (TEM). GO-MIP adsorbed twice the amount of NEO compared to its nonimprinted counterpart, and showed good NEO selectivity when under the effect of analog compound 6-biopterin (BIO). The adsorption mechanism and kinetics were best described using Freundlich isotherm and Lagergren pseudo-second-order, respectively. The adsorption capacity at equilibrium was found to be $0.4749 \mathrm{mg} / \mathrm{g}$ with the adsorption parameters as described (10 mg GO-MIP, $1 \mathrm{~mL}$ NEO 10 ppm).
\end{abstract}

Keyword: Neopterin; Graphene oxide; Molecularly imprinted polymer; Adsorption 